\title{
The accuracy of surrogate decision makers: informed consent in hypothetical acute stroke scenarios
}

\author{
Jessica Bryant ${ }^{1}$, Lesli E Skolarus², Barbara Smith ${ }^{3}$, Eric E Adelman² and William J Meurer ${ }^{2,3^{*}}$
}

\begin{abstract}
Background: Over one third of stroke patients have cognitive or language deficits such that they require surrogate consent for acute stroke treatment or enrollment into acute stroke trials. Little is known about the agreement of stroke patients and surrogates in this time-sensitive decision-making process. We sought to determine patient and surrogate agreement in 4 hypothetical acute stroke scenarios.

Methods: We performed face to face interviews with ED patients at an academic teaching hospital from June to August 2011. Patients and the surrogates they designated were asked to make decisions regarding 4 hypothetical stroke scenarios: 2 were treatment decisions; 2 involved enrollment into a clinical trial. Percent agreement was calculated as measures of surrogate predictive ability.

Results: A total of 200 patient/surrogate pairs were interviewed. Overall patient/surrogate percent agreement was $76.5 \%$. Agreement for clinical scenarios ranged from $87 \%$ to $96 \%$ but dropped to $49 \%-74 \%$ for research scenarios.

Conclusions: Surrogates accurately predict patient preferences for standard acute stroke treatments. However, the accuracy decreases when predicting research participation suggesting that the degree of surrogate agreement is dependent on the type of decision being made. Further research is needed to more thoroughly characterize surrogate decision-making in acute stroke situations.
\end{abstract}

Keywords: Acute stroke, Cerebrovascular accident, Drug trials, Emergency medicine, Stroke care, Thrombolysis, TPA, Surrogate consent

\section{Background}

By its nature, acute stroke treatment often involves cognitively-impaired-patients who need to make urgent decisions regarding high-risk-treatments within a short window of time. In fact, over $35 \%$ of stroke patients have acute cognitive or language difficulties that prohibit them from consenting to emergency acute stroke treatments [1]. Thus, acute stroke treatment decisions are often made by surrogate decision-makers (patient proxies), who are typically family members. Surrogate decision-making based on "substituted judgment" - the idea that surrogates choose the treatment the patient would most want to

\footnotetext{
* Correspondence: wmeurer@med.umich.edu

${ }^{2}$ Department of Neurology, University of Michigan, Ann Arbor, MI 48109, USA

${ }^{3}$ Department of Emergency Medicine, University of Michigan, Taubman

Center B1354 SPC 5303, 1500 E. Medical Center Drive, Ann Arbor,

Ml 48109, USA

Full list of author information is available at the end of the article
}

receive-is commonly employed in many fields of medicine to justify the treatment decisions that are made on behalf of incapacitated patients. Utilizing substituted judgment is a means by which medical professionals attempt to observe the ethical principle of respect for persons and patient autonomy in decision making.

Several studies have assessed the ability of surrogates to accurately predict patients' preferences regarding both research participation $[2,3]$ and the type of treatment in the critical care setting [4-8]. A systematic review revealed that surrogates predict ICU patients' treatment preferences with only $68 \%$ accuracy [9]. Both patient designation of surrogates and prior surrogate-patient discussion of patients' preferences failed to improve surrogates' accuracy in predicting ICU patient desires [9]. Previous work on the accuracy of surrogates in predicting patients' consent for research in the intensive care setting found false-positive

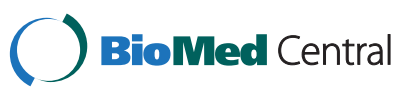


(surrogate consenting when patient would not have) consent rates for both a low-risk and a high-risk study to be $16 \%$ and $20.3 \%$ respectively [3]. Other studies investigating surrogate ability to predict participation in clinical research scenarios found that surrogates frequently made decisions based on their own preferences and refused research participation by proxy when it was in fact desired $[2,10]$. Given significant observed discrepancies between the choices of surrogates and patients, controversy exists as to the importance, validity, and ethical integrity of obtaining surrogate consent for incapacitated patients [11-13].

Given the time-sensitive nature of the condition, surrogate consent for both treatment and research options on behalf of cognitively-impaired-patients with acute stroke is currently routine practice. The impact of surrogate consent within the acute stroke setting has not previously been investigated. We sought to determine the level of patient-surrogate agreement given hypothetical standard and experimental stroke treatment scenarios.

\section{Methods \\ Setting}

We performed cross-sectional surveys via face-to-face interviews of patients and their self-designated surrogates in the University of Michigan Hospital Emergency Department (ED) between June and August 2011.

\section{Participant identification}

Patients were screened in the ED based on chief complaint and vital signs obtained from the ED electronic information system. Adult patients (ages 18 and over) were eligible for inclusion if they presented to the ED without a diagnosis of stroke or altered mental status, and with stable vital signs. In addition, participants had to be accompanied by a family member, friend, or significant other who might act as the surrogate decision maker in a real life setting.

\section{Scenarios}

The scenarios were developed and then pilot-tested using semi-structured interviews in a small group of healthy, community dwelling individuals. Feedback regarding scenario wording and clarity informed adjustments to the scenarios and questions. Briefly, scenarios 1 and 2 asked the participants a treatment question only with no research element. Specifically, scenario 1 presented a choice between IV tPA and no treatment for stroke, while scenario 2 presented the treatment options of IV tPA or an endovascular clot removal procedure.

Scenarios 3 and 4 involved deciding between standard IV tPA and an experimental treatment within the context of a research trial. Scenario 4 was identical to scenario 3, except randomization in scenario 3 was fixed at 50:50 and response adaptive randomization was described for scenario 4. Response adaptive randomization changes the allocation ratio based on information accumulated during the trial to randomize more patients to whichever arm is performing better (possibly the active treatment or the control) [14]. The exact wording of the scenarios is available in Additional file 1.

\section{Interview procedures}

After verbal informed consent was obtained, the surrogate was asked to leave the area. The patient was first asked if he/she could identify any of the warning signs of stroke, in order to assess his/her current knowledge of stroke symptoms [15]. Adequate stroke knowledge was defined as the ability to name two of five acute stroke symptoms (headache, paralysis, trouble speaking/confusion, vision changes, and dizziness). The patient was then presented with the four scenarios for decisions in the event of an acute stroke. The scenarios and data collection instrument are available as a web appendix.

After each scenario, the designated patient was asked to indicate his or her preferred treatment or research choice between the two offered. He/she was then presented with a 10-point Likert scale and asked to indicate his/her confidence in that decision - that he or she had made the right choice $(0=$ not at all confident, $10=$ absolutely confident $)$. The same scenarios were then presented to the surrogates, in the same order and with identical wording. Additionally, surrogates were asked to indicate their certainty that the patient made the same treatment or research choice. Again, a 10-point Likert scale was used for assessments $(0=$ completely unsure, $10=$ completely sure $)$. Patients and surrogates were allowed to ask questions regarding the scenarios before making decisions; the study team created a set of standard answers to the most commonly asked questions after the first week of recruitment. Demographics for patients/surrogates were collected after the completion of the interviews. No protected health information or specific patient or surrogate identifiers were collected. Zip codes were collected in order to estimate household incomes of the communities in which the patients and surrogates resided, using the census.gov website [16].

\section{Statistical analysis}

The primary outcome variable was the overall weighted Cohen's kappa statistic for agreement between patient and surrogate. This was calculated for each of the four scenarios. Other collected data, including demographic information and responses to the Likert scales for confidence and certainty of decisions made was summarized using means with standard deviation or proportions as appropriate. In addition, we also calculated Gwet's AC1 which addresses the problem of a kappa statistic in low prevalence situations [17-19]. 


\section{Sample size calculation}

We hypothesized that modest agreement would occur at baseline (kappa $=0.5)$ [16]. We assumed that patients would consent to hypothetical treatments or research trials $80 \%$ of the time, and that surrogates would consent $70 \%$ of the time. Based on our assumptions and using the N.cohen.kappa function from the Concord package of $\mathrm{R}$ Version 2.8.1, we estimated 90\% power to detect a kappa statistic of 0.7 or higher with a total sample size of 200 . We doubled this sample size to be conservative as we were aware of no prior pilot data that provided estimates of the performance of surrogate consent in acute stroke research.

\section{Human subjects protection}

The study protocol was reviewed and approved by the University of Michigan Institutional Review Board and was granted Exempt status. Potential participants were provided with an information sheet describing the study and indicating that participation was completely voluntary. In addition, patients were assured that treatment for their presenting complaints would not be compromised by declining to participate. Participants were given the opportunity to have questions answered, and if they agreed, verbal consent was obtained.

\section{Results}

\section{Subject characteristics}

400 participants were enrolled in the study - 200 "patients" and 200 "surrogates." Characteristics of both are shown in Table 1. Stroke knowledge was similar between the patient and surrogate groups, with $71 \%$ and $74 \%$, respectively, of patients and surrogates able to provide at least 2 out of 5 possible stroke symptoms. Mean community household income was the same for patients and surrogates at $\$ 54,144$ and is higher than the national average of $\$ 41,994$ [16].

\section{Patient and surrogate decisions}

Overall, surrogates predicted patients' treatment preferences with $76.5 \%$ crude agreement; however, the kappa statistics for each scenario indicated poor agreement. The kappa statistics ranged from 0.18 in scenario 1 to -0.02 in scenario 4 . The full results for each scenario are presented in Table 2. In scenario 1 in which subjects were given the choice between being given tPA or undergoing no treatment, surrogates had $96 \%$ prediction accuracy; the overwhelming majority of patients and surrogates chose the treatment of tPA over no treatment. Patient/surrogate agreement in scenario 2 (clot removal procedure vs. tPA) was $87 \%$; the vast majority of agreement was found when both parties refused the procedure and opted for tPA instead.

In scenario 3 (tPA vs. standard RCT), 5\% of patient/ surrogate pairs consented to the trial while $70 \%$ of pairs
Table 1 Patient and surrogate characteristics

\begin{tabular}{|c|c|c|c|c|}
\hline \multirow[b]{2}{*}{ Characteristics } & \multicolumn{2}{|c|}{ Patients } & \multicolumn{2}{|c|}{ Surrogates } \\
\hline & $\mathrm{N}$ & $\%$ & $\mathbf{N}$ & $\%$ \\
\hline Female & 122 & $61 \%$ & 120 & $60 \%$ \\
\hline Male & 78 & $39 \%$ & 80 & $40 \%$ \\
\hline \multicolumn{5}{|l|}{ Ethnicity } \\
\hline White & 178 & $89.5 \%$ & 179 & $89.5 \%$ \\
\hline African American & 14 & $7 \%$ & 14 & $7 \%$ \\
\hline Hispanic & 2 & $1 \%$ & 2 & $1 \%$ \\
\hline Asian & 3 & $1 \%$ & 2 & $1 \%$ \\
\hline Other & 3 & $1.5 \%$ & 3 & $1.5 \%$ \\
\hline \multicolumn{5}{|l|}{ Education } \\
\hline Some high school & 14 & $7 \%$ & 8 & $4 \%$ \\
\hline High school graduate & 39 & $19.5 \%$ & 44 & $22 \%$ \\
\hline Some college & 77 & $38.5 \%$ & 61 & $30.5 \%$ \\
\hline College graduate & 44 & $22 \%$ & 53 & $26.5 \%$ \\
\hline Post graduate degree & 26 & $13 \%$ & 34 & $17 \%$ \\
\hline \multicolumn{5}{|l|}{ Medical history } \\
\hline Myocardial infarction & 15 & $7.5 \%$ & 5 & $2.5 \%$ \\
\hline Diabetes & 24 & $12 \%$ & 15 & $7.5 \%$ \\
\hline Stroke & 6 & $3 \%$ & 8 & $4 \%$ \\
\hline Hypertension & 49 & $24.5 \%$ & 44 & $22 \%$ \\
\hline Atrial fibrillation & 14 & $7 \%$ & 4 & $2 \%$ \\
\hline None of the above & 132 & $66 \%$ & 144 & $72 \%$ \\
\hline \multicolumn{5}{|c|}{ Surrogate relationship to patient } \\
\hline Spouse & & & 83 & $41.5 \%$ \\
\hline Child & & & 24 & $12 \%$ \\
\hline Parent & & & 29 & $14.5 \%$ \\
\hline Sibling & & & 10 & $5 \%$ \\
\hline Significant other & & & 21 & $10.5 \%$ \\
\hline \multirow[t]{2}{*}{ Other } & & & 28 & $14 \%$ \\
\hline & Mean & SD & Mean & SD \\
\hline Age, years & 46.4 & 18.4 & 47.2 & 16 \\
\hline Number of siblings & 2.5 & 1.8 & 2.4 & 1.9 \\
\hline Median household income & $\$ 54,144$ & $\$ 14,818$ & $\$ 54,144$ & $\$ 13,464$ \\
\hline
\end{tabular}

refused the trial, resulting in an agreement rate of $74 \%$. The majority of disagreement (65\%) was found when patients desired the trial but proxies refused. Lastly, scenario 4 (tPA vs. adaptive RCT) represented the lowest rate of surrogate/proxy agreement at $49 \%$, (pairs consenting to trial $28.5 \%$, pairs refusing trial $20.5 \%$ ) although there was higher overall consent to the trial in patients (56\%) and proxies (50\%) when compared to the standard RCT. Sensitivity and specificity were both $49 \%$.

Patients and surrogates all indicated relatively high degrees of confidence (mean confidence scores for each scenario ranged from 7.8 to 8.5 on a 10-point Likert scale) 
Table 2 Agreement between patients and surrogates for hypothetical stroke scenarios

\begin{tabular}{|c|c|c|c|c|c|c|c|c|c|c|c|c|c|c|c|}
\hline \multicolumn{4}{|c|}{ Scenario 1: TPA vs. no treatment } & \multicolumn{4}{|c|}{ Scenario 2: TPA vs. clot removal procedure } & \multicolumn{4}{|c|}{ Scenario 3: TPA vs. standard RCT } & \multicolumn{4}{|c|}{ Scenario 4: TPA vs. adaptive RCT } \\
\hline & \multicolumn{3}{|c|}{ Patients } & \multicolumn{4}{|c|}{ Patients } & \multicolumn{4}{|c|}{$\begin{array}{l}\text { Patients } \\
\end{array}$} & \multicolumn{4}{|c|}{$\begin{array}{l}\text { Patients } \\
\end{array}$} \\
\hline \multirow{4}{*}{ Proxies } & $\begin{array}{l}\text { Would you } \\
\text { want TPA? }\end{array}$ & Yes & No & & $\begin{array}{l}\text { Would you want } \\
\text { the procedure? }\end{array}$ & Yes & No & \multirow{3}{*}{ Proxies } & $\begin{array}{c}\text { Would you } \\
\text { want the trial? }\end{array}$ & Yes & No & \multirow{3}{*}{ Proxies } & $\begin{array}{l}\text { Would you } \\
\text { want the trial? }\end{array}$ & Yes & No \\
\hline & Yes & $\begin{array}{c}95.5 \% \\
(n=191)\end{array}$ & $\begin{array}{c}2 \% \\
(n=4)\end{array}$ & Proxies & Yes & $\begin{array}{c}1 \% \\
(n=2)\end{array}$ & $\begin{array}{c}4.5 \% \\
(n=9)\end{array}$ & & Yes & $\begin{array}{c}4.5 \% \\
(n=9)\end{array}$ & $\begin{array}{c}9 \% \\
(n=18) \\
\end{array}$ & & Yes & $\begin{array}{c}28.5 \% \\
(n=57) \\
\end{array}$ & $\begin{array}{c}21.5 \% \\
(n=43) \\
\end{array}$ \\
\hline & \multirow[t]{2}{*}{ No } & $\begin{array}{c}2 \% \\
(n=4)\end{array}$ & $\begin{array}{l}0.5 \% \\
(n=1)\end{array}$ & & No & $\begin{array}{c}8.5 \% \\
(n=17)\end{array}$ & $\begin{array}{c}86 \% \\
(n=172)\end{array}$ & & No & $\begin{array}{c}17 \% \\
(n=34)\end{array}$ & $\begin{array}{c}69.5 \% \\
(n=139)\end{array}$ & & No & $\begin{array}{l}29.5 \% \\
(n=59)\end{array}$ & $\begin{array}{c}20.5 \% \\
(n=41)\end{array}$ \\
\hline & & Mean & SD & & & Mean & SD & & & Mean & SD & & & Mean & SD \\
\hline \multicolumn{2}{|c|}{ Patient confidence } & 8.0 & 2.1 & \multicolumn{2}{|c|}{ Patient confidence } & 8.1 & 2 & \multicolumn{2}{|c|}{ Patient confidence } & 8.0 & 1.9 & \multicolumn{2}{|c|}{ Patient confidence } & 7.8 & 1.9 \\
\hline \multicolumn{2}{|c|}{ Proxy confidence } & 8.5 & 1.7 & \multicolumn{2}{|c|}{ Proxy confidence } & 8.5 & 1.9 & \multicolumn{2}{|c|}{ Proxy confidence } & 8.4 & 1.9 & \multicolumn{2}{|c|}{ Proxy confidence } & 8.0 & 1.9 \\
\hline \multicolumn{2}{|c|}{ Proxy certainty } & 8.4 & 1.9 & \multicolumn{2}{|c|}{ Proxy certainty } & 8.0 & 2.2 & \multicolumn{2}{|c|}{ Proxy certainty } & 7.8 & 2.3 & \multicolumn{2}{|c|}{ Proxy certainty } & 7.5 & 2.1 \\
\hline \multicolumn{2}{|c|}{$\begin{array}{c}\text { Cohen's } \\
\text { Kappa statistic }\end{array}$} & \multicolumn{2}{|c|}{0.18} & \multicolumn{2}{|c|}{$\begin{array}{c}\text { Cohen's } \\
\text { Kappa statistic }\end{array}$} & \multicolumn{2}{|c|}{0.07} & \multicolumn{2}{|c|}{$\begin{array}{c}\text { Cohen's } \\
\text { Kappa statistic }\end{array}$} & \multicolumn{2}{|c|}{0.11} & Kap & $\begin{array}{l}\text { Ohen's } \\
\text { pa statistic }\end{array}$ & \multicolumn{2}{|c|}{-0.02} \\
\hline \multicolumn{2}{|c|}{ AC1 (95\% Cl) } & \multicolumn{2}{|c|}{$0.96(0.93-0.99)$} & \multicolumn{2}{|c|}{ AC1 (95\% Cl) } & \multicolumn{2}{|c|}{$0.87(0.79-0.91)$} & \multicolumn{2}{|c|}{ AC1 $(95 \%$ Cl) } & $0.63(0$ & $.53-0.74)$ & & (95\% Cl) & $-0.01(-0$ & $13-0.13)$ \\
\hline
\end{tabular}

Overall agreement is observed in the bolded diagonal cells (i.e for scenario 1: $96 \%$ of patients and proxies agreed). Counts and proportions listed for all scenarios. Patient and surrogate confidence was measured on a 10 point Likert scale. $\mathrm{SD}=$ standard deviation. $\mathrm{tPA}=$ tissue plasminogen activator. $\mathrm{RCT}=$ Randomized controlled trial. $\mathrm{AC1}=\mathrm{Gwet}$ agreement coefficient. $\mathrm{Cl}=$ confidence interval. 
in the decisions they were making. In addition, surrogates indicated that they were reasonably certain (mean certainty ranged from 7.5 to 8.4 ) that their decisions would be in concordance with the patients' wishes.

\section{Discussion and conclusions}

Our study of 200 patient/surrogate pairs found substantially more patient/surrogate agreement in scenarios involving standard treatments (scenarios 1 and 2) than for research protocols (scenarios 3 and 4). The varying predictive accuracy of surrogate decision-makers demonstrated in other studies raises questions about the ethics and validity of charging surrogates with important medical decisions, especially in an acute setting such as stroke $[4,11]$. Some ethicists argue against the use of surrogates as the decisional authority for incapacitated patients and recommend instead that surrogates serve as advisors to the medical team, who will carry out "best-interests judgment" on behalf of the patient; this is less clear regarding research participation [13]. The results of the current investigation suggest surrogates predict patient preferences for standard treatments well and perform less well when trying to predict patient preferences for research participation. It is likely that the degree of surrogate agreement is highly dependent on the type of decision being made.

When given a choice, the majority of patients and surrogates opted for standard treatments (tPA) over more experimental (scenario 2) or clinical trial (scenarios 3 and 4) alternatives. Possible explanations for this result could be risk aversion given the severe nature of the described stroke and/or time pressure to make potentially life and death decisions, leading participants to "stick with the standard" rather than choosing a newer, riskier, and often more complex alternative that would have required more thought $[2,10]$. Furthermore, the nature of our interview study limited the amount of information we could give participants regarding treatments and trials; participants may have opted for more standard treatments simply because they felt that not enough information was provided for the alternative research treatments. Our results echo the findings of several other studies in that surrogates predicted more frequently on the conservative side, (e.g., refusing research trial participation) when the patients themselves would have chosen (potentially riskier) research over standard treatment $[2,10]$.

The clinical trial featuring response adaptive randomization (scenario 4) was more often agreed to by both patients and surrogates than the standard clinical trial (scenario 3 ), although there was substantially more disagreement regarding participation in the adaptive trial. It is possible that the complexity of the adaptive trial, confusion regarding its setup, and difficulty explaining it could have contributed to the observed discrepancy. Interestingly, scenario 4 represented the lowest rate of patient/surrogate agreement and also represented the lowest confidence in decision ratings from both patients and proxies. Proxy certainty that their decision was in accordance with the patient's decision was also lowest in scenario 4 in comparison to the other scenarios. This suggests that patients and proxies are to some degree calibrated regarding the validity of their decisions, and that lack of proxy certainty is correlated with poor predictive accuracy. The therapeutic misconception (potential research volunteer belief that a research protocol confers medical benefit) has previously been described as a concern that response adaptive randomization may help mitigate [20]; we did observe numerically higher participation in the RAR scenario versus the fixed randomization design.

We recognize several limitations of our study. The precision of our estimates of agreement via the kappa statistic was limited, mainly because of the high prevalence of acceptance of standard treatments and the low prevalence of acceptance of research protocols by both patients and surrogates. This phenomenon is known as the "kappa paradox" and occurs in settings where the prevalence of a "yes" response is very high (i.e., consenting to the standard treatment of tPA) or very low (i.e., refusing participation in the research trials) [21]. The high or low prevalence of "yes" responses results in a high likelihood that agreement between surrogates and patients occurred by chance alone-a point that when taken into account by kappa results in low kappa statistics despite high rates of agreement between groups. For this reason, the Cohen kappa statistics here do not adequately reflect the degree of agreement between patients and surrogate decisionmakers. Our calculations of the AC1 may provide a more meaningful picture, indicating fairly good to excellent agreement on scenarios 1 to 3 . Another limitation is that our secondary measurements of certainty and confidence were on the Likert scale, and required that participants make probability estimations by choosing from ordinal numbers from 0 to 10 . An additional limitation is that hypothetical scenarios raised many questions from subjects that likely caused participants to receive more, less, or different information than others. Although we developed a set of answers to commonly asked questions early in the study in an effort to standardize information as much as possible, it is not possible to anticipate every question for every discrete treatment or trial. In fact, this reflects the "real world" consent process. Furthermore, participants were not provided with an option for "undecided." This undoubtedly forced participants into decisions they were not comfortable with or did not fully understand. However, this is identical to what happens in the setting of actual acute stroke. Finally, since these scenarios were hypothetical, it is unclear how our research would compare to patient/surrogate decisions in actual acute stroke presentations. 
As to the generalizability of this study, the population was recruited from the area surrounding the University of Michigan Medical Center, and was therefore on average more educated, predominately Caucasian, and lived in areas of higher median income than the overall US population. Thus, the results of this study may not be generalizable to other, more urban or rural medical centers. Furthermore, patient/surrogate pairs were younger than typical stroke patient/surrogate pairs, potentially leading to results that do not reflect the preferences of the actual stroke victims and their surrogates in clinical practice. This is important to consider as the young may discount the quality of life of older adults - therefore adult children may act differently as surrogates decision makers compared to spouses/ significant others.

In summary, patient/surrogate agreement varies depending on the type of clinical or research decision being made. Future research might investigate how patient/surrogate agreement compares to patient/physician agreement as medical providers are the most likely alternative decision makers when surrogates cannot be identified. An additional important area for future research is the degree to which potential research subjects understand the trial designs. Further research designs may benefit from a mixed methods approach to explore in more detail the nature of patient/ surrogate discrepancies in order to develop a conceptual model for surrogate decision-making in acute settings.

\section{Additional file}

Additional file 1: Full text of hypothetical scenarios presented to research subjects.

\section{Competing interests}

The authors declare that they have no competing interests.

\section{Authors' contributions}

$\mathrm{JB}$ was responsible for acquisition of the data, interpretation of the data, drafting of the manuscript, and administrative, technical and material support. WM provided study concept and design, analysis and interpretation of the data, revision of the manuscript, and study supervision. LS and EA aided in interpretation of the data and manuscript revision. BS provided administrative, technical and material support. All authors read and approved the final manuscript.

\section{Author details}

${ }^{1}$ University of Michigan, Medical School, Ann Arbor, Ml 48109, USA.

Department of Neurology, University of Michigan, Ann Arbor, MI 48109, USA. ${ }^{3}$ Department of Emergency Medicine, University of Michigan, Taubman Center B1354 SPC 5303, 1500 E. Medical Center Drive, Ann Arbor, Ml 48109, USA.

Received: 1 February 2013 Accepted: 5 November 2013

Published: 13 November 2013

\section{References}

1. Pedersen PM, Stig Jørgensen $H$, Nakayama $H$, Raaschou HO, Olsen TS: Aphasia in acute stroke: incidence, determinants, and recovery. Ann Neurol 1995, 38(4):659-666.
2. Muncie HL, Magaziner J, Hebel JR, Warren JW: Proxies' decisions about clinical research participation for their charges. J Am Geriatr Soc 1997, 45(8):929-933.

3. Coppolino M, Ackerson L: Do surrogate decision makers provide accurate consent for intensive care research? Chest 2001, 119(2):603-612.

4. Sachs GA, Stocking CB, Stern R, Cox DM, Hougham G, Sachs RS: Ethical aspects of dementia research - informed consent and proxy consent. Clin Res 1994, 42(3):403-412.

5. Sulmasy DP, Haller K, Terry PB: More talk, less paper - predicting the accuracy of substituted judgments. Am J Med 1994, 96(5):432-438.

6. Suhl J, Simons P, Reedy T, Garrick T: Myth of substituted judgment - surrogate decision-making regarding life-support is unreliable. Arch Intern Med 1994, 154(1):90-96.

7. Uhlmann RF, Pearlman RA, Cain KC: Physicians and spouses predictions of elderly patients resuscitation preferences. J Gerontol 1988, 43(5):M115-M121.

8. Hare J, Pratt C, Nelson C: Agreement between patients and their self-selected surrogates on difficult medical decisions. Arch Intern Med 1992, 152(5):1049-1054.

9. Shalowitz DI, Garrett-Mayer E, Wendler D: The accuracy of surrogate decision makers: a systematic review. Arch Intern Med 2006, 166(5):493-497.

10. Kasner SE, Del Giudice A, Rosenberg S, et al: Who will participate in acute stroke trials? Neurology 2009, 72(19):1682-1688.

11. Leira EC, Kaldjian LC, Ludwig BR, et al: Lack of international consensus on ethical aspects of acute stroke trials. J Stroke Cerebrovasc Dis 2012, 21(3):200-204

12. Flaherty ML, Karlawish J, Khoury JC, Kleindorfer D, Woo D, Broderick JP: How important is surrogate consent for stroke research? Neurology 2008, 71(20):1566-1571.

13. Wrigley A: Proxy consent: moral authority misconceived. $J$ Med Ethics 2007, 33(9):527-531.

14. Berry DA: Adaptive clinical trials: the promise and the caution. J Clin Oncol 2011, 29(6):606-609.

15. Silver FL, Rubini F, Black D, Hodgson CS: Advertising strategies to increase public knowledge of the warning signs of stroke. Stroke 2003, 34(8):1965-1968.

16. Landis JR, Koch GG: The measurement of observer agreement for categorical data. Biometrics 1977, 33(1):159-174.

17. Brasil PEAAd: Gwet's AC1 Interrater Reliability; 2012. http://permalink.gmane. org/gmane.comp.lang.r.epi/130. Accessed 9/24/2013, 2013.

18. Gwet KL: Computing inter-rater reliability and its variance in the presence of high agreement. Br J Math Stat Psychol 2008, 61(1):29-48.

19. Wongpakaran N, Wongpakaran T, Wedding D, Gwet K: A comparison of Cohen's kappa and Gwet's AC1 when calculating inter-rater reliability coefficients: a study conducted with personality disorder samples. BMC Med Res Methodol 2013, 13(1):61.

20. Meurer WJ, Lewis RJ, Berry DA: Adaptive clinical trials: a partial remedy for the therapeutic misconception? JAMA 2012, 307(22):2377-2378.

21. Viera AJ, Garrett JM: Understanding interobserver agreement: the kappa statistic. Fam Med 2005, 37(5):360-363.

doi:10.1186/1471-227X-13-18

Cite this article as: Bryant et al:: The accuracy of surrogate decision makers: informed consent in hypothetical acute stroke scenarios. BMC Emergency Medicine 2013 13:18.

\section{Submit your next manuscript to BioMed Central and take full advantage of:}

- Convenient online submission

- Thorough peer review

- No space constraints or color figure charges

- Immediate publication on acceptance

- Inclusion in PubMed, CAS, Scopus and Google Scholar

- Research which is freely available for redistribution 